\title{
Special issue introduction: e-future
}

\author{
Nilmini Wickramasinghe • Douglas Vogel
}

Published online: 26 May 2012

(C) IUPESM and Springer-Verlag 2012

Without exception, all countries of the OECD are experiencing exponentially increasing healthcare costs [1]. Most have agreed that technology and more specifically e-health holds the key [2]. Although many definitions exist, e-health essentially involves the application of ICTs (information communication technologies) to support and facilitate the range of healthcare functions concerned with the practice and delivery of care [3-6]. Moreover, e-health; also includes the digitising of various healthcare processes and tasks including e-billing, e-payment, e-prescription, e-radiology and e-records [6]. What becomes important then is to understand the key challenges, barriers and facilitators when designing e-health solutions so that they will indeed serve to realize a healthcare value proposition of excellence in access, quality and value and serve to ensure a better future for healthcare delivery. This, in turn, requires consideration of the key future trends that will significantly impact healthcare [2], namely:

(i) escalating healthcare costs

(ii) empowered consumers

(iii) e-health adaptability

(iv) a shift to focus on healthcare prevention as well as cure

(v) an increase in non-communicable (chronic) diseases

(vi) an aging population

N. Wickramasinghe $(\bowtie)$

Epworth Chair Health Information Management,

HIRi \& SBITL, RMIT University,

GPO Box 2476, Melbourne, VIC 3001, Australia

e-mail: nilmini.work@gmail.com

D. Vogel

Information Systems, City University of Hong Kong,

G7315, Academic 1,

Hong Kong, China

e-mail: isdoug@cityu.edu.hk (vii) impact on health insurance

(iix) workforce shortages

(ix) the role of data and information

(x) the importance of security and privacy

In order to illustrate possible solutions to address these challenges from different parts of the world, and thereby also highlight appropriate facilitators and potential barriers, we have compiled this collection of four papers which discuss key topics within this broad area.

Paper 1 entitled "The LITIS conceptual framework: measuring eHealth readiness and adoption dynamics across the Healthcare Organizations" by Tamburis et al. presents a discussion of a framework that entails a taxonomy of indicators to assess the eHealth readiness and adoption in the context of Italy.

In paper 2, Heiro and Mäntymäki in their paper "Managing medication information with electronic patient records-a Finnish clinicians' perspective" discuss an example from Finland.

Paper 3 "Risk evaluation and security analysis of the clinical area within the German electronic health information system" is by Sunyaev and Pflug.

Finally, Paper 4 by Yin et al. "Examining user satisfaction of school nursing information system in Taiwan" provides an interesting e-health example that focuses on nursing from Taiwan.

We hope our readers will enjoy this journey into the possibilities for e-health as well as find useful insights and ideas for future research into various macro- and micro-level issues that, when resolved and taken together, will enable superior healthcare delivery.

The Special Issue Editors

Nilmini Wickramasinghe \& Doug Vogel 


\section{References}

1. World Healthcare Organization. 2008. http://www.who.int/en/ (accessed 10th Oct 2011).

2. Wickramasinghe N, Schaffer J. Realizing value driven e-health solutions. Report for IBM. Washington DC; 2010.

3. Varshney U. Pervasive Healthcare and Wireless Health monitoring. Mobile Net Appl. 2007;12:113-27.
4. Varshney U. Pervasive healthcare computing. New York: Springer; 2009.

5. Zwicker M, Seitz J, Wickramasinghe N. In: The telematics infrastructure: the backbone of the german e-health card. Int J Healthcare Delivery Reform. 2011 (in press).

6. Wickramasinghe N. Pervasive computing and healthcare. In: Bali et al., editors. Pervasive KM for healthcare. New York: Springer; 2011 (in press). 